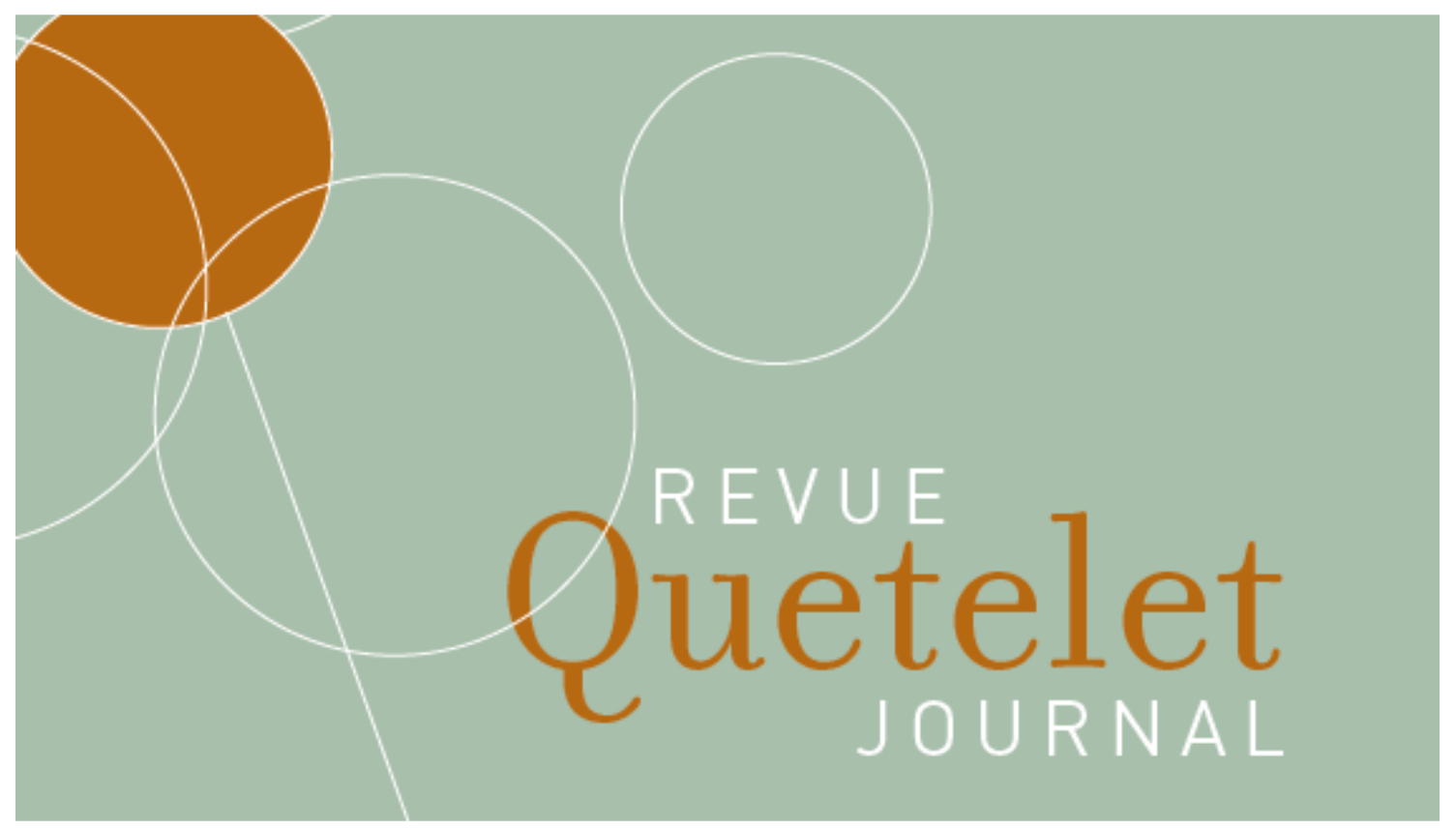

Vol. 5, n¹, avril 2017, pp. 99-125

DOI : 10.14428/rqj2017.05.01.04

ISSN: 20349378

\title{
Vieillissement de la population et politiques publiques réunionnaises à l'échelle infra-territoriale
}

Armelle Klein, Frédéric Sandron

C2017 Armelle Klein, Frédéric Sandron

This work is licensed under a Creative Commons Attribution-NonCommercial 4.0 International License. You can share, adapt the material for non-commercial purposes provided that you give appropriate credit and indicate if changes were made. For details see https://creativecommons.org/licenses/by-nc/4.0/

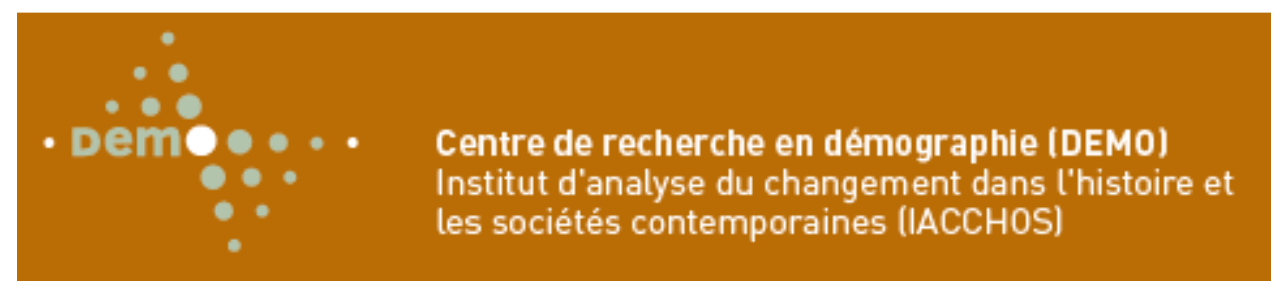





\title{
Vieillissement de la population et politiques publiques réunionnaises à l'échelle infra-territoriale
}

\author{
ARMELLE KLEIN ${ }^{1}$ \\ FRÉDÉRIC SANDRON ${ }^{2}$
}

\begin{abstract}
Résumé
L'île de La Réunion est un département français d'outre-mer situé dans le SudOuest de l'océan Indien, peuplé d'environ 850000 personnes. La forte diminution de la fécondité entre les années 1960 et 1980 entraîne un vieillissement de la population réunionnaise extrêmement rapide, puisque la proportion de personnes âgées devrait doubler entre 2010 et 2030 . Ce constat général masque cependant des différences infra-territoriales importantes, que ce soit en termes de proportion de personnes âgées par commune ou de la proportion des personnes âgées qui perçoivent une aide sociale liée à la perte d'autonomie. Si l'on ajoute à ces facteurs la géographie très accidentée de ce territoire montagneux et les inégalités d'accès aux services de santé, on perçoit mieux la nécessité de décliner à un échelon infraterritorial la problématique de l'aménagement du territoire face au vieillissement de la population.
\end{abstract}

\section{Mots-clés}

Vieillissement, politiques publiques, territoire, La Réunion, inégalités.

\begin{abstract}
Reunion Island is a French overseas department in the Southwest Indian Ocean, populated by about $850^{\prime} 000$ people. The sharp decline in fertility between the 1960 s and the 1980s led to an extremely rapid aging of the population of Reunion Island. The share of people over the age of 60 is expected to double between 2010 and 2030. However, at a smaller scale, this general observation masks territorial differences like the share of old people in the population per commune or the share of old people receiving social assistance related to the loss of autonomy. In addition to these factors, the very harsh landscape of this mountainous territory
\end{abstract}

1. Université de La Réunion, Laboratoire LCF, IRD, PEBCS, La Réunion.

2. Institut de Recherche pour le Développement (IRD), CEPED, La Réunion. 
and inequalities of access to health services, encourage us to deal with the problem of spatial planning and aging of the population at a sub-territorial level.

\section{Keywords}

Aging, public policies, territory, Reunion Island, Inequalities.

\section{Introduction}

L'île de La Réunion est un département français d'outre-mer situé dans le Sud-Ouest de l'océan Indien. D'une superficie de $2500 \mathrm{~km}^{2}$, il est peuplé d'environ 850000 personnes en 2015 réparties dans 24 communes. Si la population reste jeune du fait d'une fécondité qui stagne depuis une vingtaine d'années à environ 2,4 enfants par femme, la baisse très rapide de cette dernière entre les années 1960 et 1980 et l'augmentation de l'espérance de vie entraînent cependant un vieillissement de la population extrêmement rapide (Figures 1 et 2). Ainsi, la proportion de personnes âgées de soixante ans et plus dans la population totale devrait doubler entre 2010 et 2030 (Insee, 2014a). Ce vieillissement de la population réunionnaise et les défis et enjeux qu'il pose aux collectivités publiques s'inscrivent dans une réflexion nationale dans le cadre du Projet de loi relatif à l'adaptation de la société au vieillissement dont la mise en application est prévue au 1er janvier 2016.

FIGURE 1 Indice synthétique de fécondité à La Réunion

et en France métropolitaine (1953-2013)

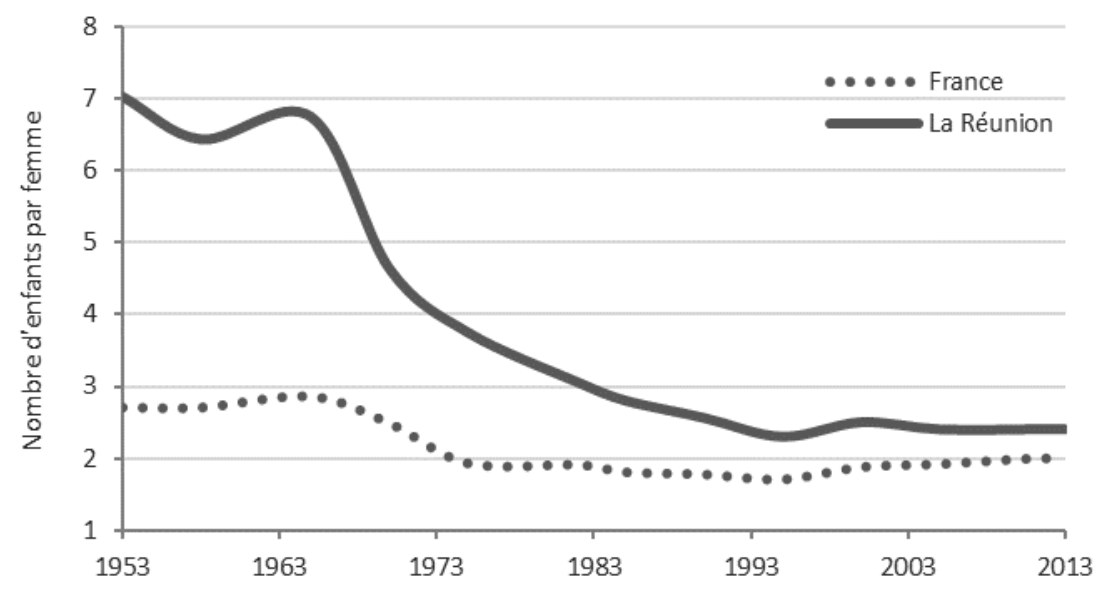

Source : Insee. 
Figure 2 Espérance de vie à La Réunion

et en France métropolitaine (1953-2013)

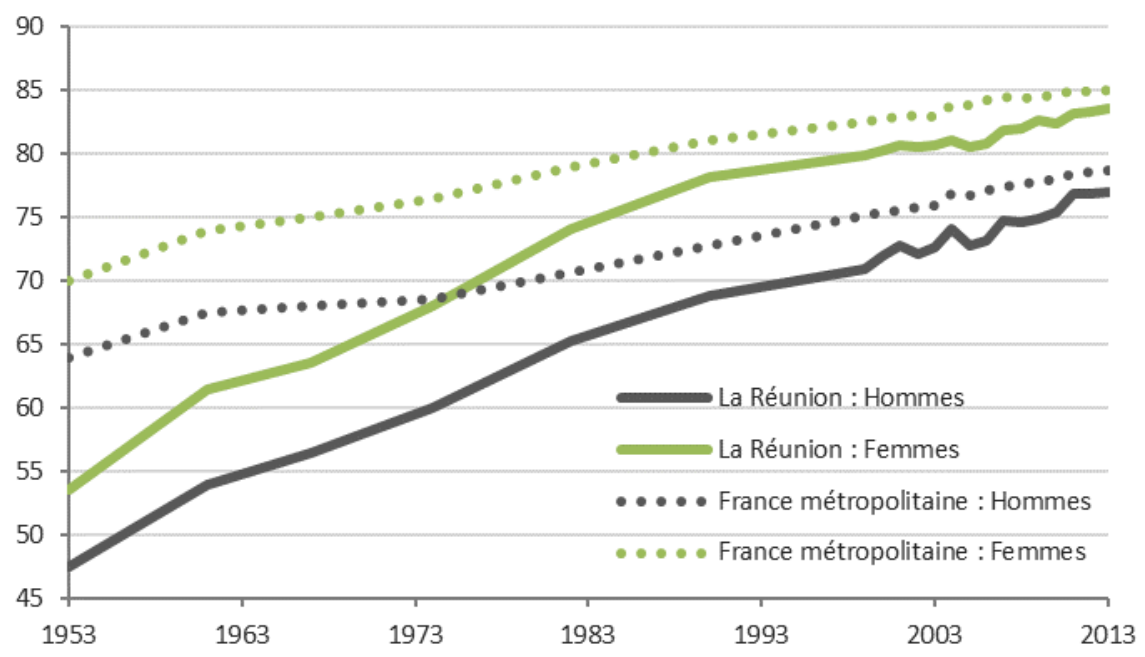

Source : Insee, État civil in Insee (2015).

Dans les orientations récentes des politiques publiques françaises, mais aussi européennes, figure une importance croissante donnée à la notion de territoire (Berrut, Guérin, 2014). Au-delà des grandes orientations stratégiques, la compréhension fine des phénomènes démographiques et sociaux se situe à une échelle infra-départementale. Ceci est particulièrement vrai dans un département comme La Réunion qui connaît une forte hétérogénéité en termes de précarité et de vulnérabilité (Insee, 2013a). Par exemple, à l'échelle communale, la proportion de personnes âgées qui perçoivent l'Allocation Minimum Vieillesse varie de 34,5\% à 60,7 \% en 2008 (Insee, 2011a). Si l'on ajoute à ces considérations démographiques et économiques une géographie très accidentée de ce territoire montagneux et le constat de l'existence de logements souvent vétustes pour les personnes âgées (Insee, 2010), on perçoit l'importance de décliner la problématique de l'aménagement du territoire face au vieillissement de la population à un échelon infra-départemental.

La question qui nous intéresse ici est donc de comprendre comment les politiques publiques s'adaptent au vieillissement de la population et aux spécificités des personnes âgées concernées sur le territoire réunionnais. Dans cette perspective, une première section dressera un rapide bilan des caractéristiques territoriales et infra-territoriales de lîle de La Réunion. Une deuxième section recentrera le propos sur le vieillissement démographique à l'échelle communale. Dans une troisième sec- 
tion, la distribution et la répartition de l'offre sanitaire et sociale seront elles aussi étudiées selon cet échelon territorial. La quatrième et dernière section s'intéressera à un panel d'initiatives publiques illustrant la nécessité d'une approche fine et infra-départementale en matière de services sanitaires et sociaux pour les personnes âgées.

\section{Un territoire socio-économiquement hétérogène}

Peuplée en 1663 et orientée durant la majeure partie de son histoire vers l'agriculture de rente, c'est en 1946 que l'île de La Réunion devient un département français de l'outre-mer. À cette date, le niveau de développement économique y est extrêmement faible. Les deux tiers de la population active sont employés dans le secteur agricole, seulement un logement sur dix est équipé en eau courante ou en électricité, l'urbanisation ne concerne que $20 \%$ de la population, l'espérance de vie est de 48 ans et le taux de mortalité infantile de $160 \%$ (Sandron, 2007).

À partir de la seconde moitié du vingtième siècle, et plus précisément à partir de la décennie 1960, les politiques publiques en matière de création d'infrastructures et d'organisation administrative, telles les routes, les écoles, les hôpitaux, la sécurité sociale, l'électrification ou encore l'adduction d'eau potable, ont été à l'origine d'un rattrapage dans les domaines économique et social de La Réunion par rapport à la métropole française. En quelques décennies, l'île de La Réunion a globalement rejoint les standards européens, même si les modalités de la croissance et la trajectoire du développement économique en font un département atypique dans le paysage français.

En effet, avec une croissance annuelle moyenne du PIB en volume de l'ordre de $5 \%$ entre les années 1970 et le début des années 2000, les taux de croissance réunionnais sont, sur le long terme, bien supérieurs à ceux enregistrés en métropole (Rochoux, 2004). Cependant, cette dynamique a priori positive s'est accompagnée d'une montée concomitante du taux de chômage qui se situait aux alentours de $40 \%$ en 2000 . Au cours de la décennie 2000, la croissance du PIB réunionnais a continué de présenter des niveaux supérieurs à ceux de la métropole, mais toujours assortie d'un chômage élevé avec des taux de l'ordre de $30 \%$ au début de la décennie 2010. Cette situation de chômage récurrent est similaire dans l'ensemble des économies ultramarines françaises (L'Horty, 2014). 
Une autre spécificité que nous devrons prendre en compte dans notre analyse est liée à un ensemble de contraintes que connaissent la plupart des «petits territoires insulaires» ou des «petites économies insulaires». Selon Briguglio (1995), il existe pour ces territoires de nombreux facteurs de vulnérabilité parmi lesquels, l'étroitesse du marché domestique, la grande dépendance à l'importation, le faible éventail de production locale, la non maîtrise de la structure des prix à l'import-export, l'absence d'économies d'échelle, les prix élevés liés au coût de transport des marchandises, l'isolement géographique, l'exposition aux risques naturels, le peu de ressources naturelles et la forte émigration.

Exposée aux cyclones, éloignée des grands marchés mondiaux et de la métropole française, présentant une structure de production économique peu diversifiée et faisant face à un marché intérieur inférieur à un million de consommateurs, La Réunion est clairement un territoire «vulnérable» (Goavec, Hoarau, 2015). Parmi les facteurs de vulnérabilité, la configuration spatiale du territoire de La Réunion, île volcanique et montagneuse, induit une concentration de l'habitat à $80 \%$ sur les côtes. La politique de développement impulsée à La Réunion dans les années 1950 a de surcroît favorisé cette littoralisation de l'habitat résidentiel, à tel point qu'en 1975, un Plan à l'Aménagement des Hauts a été créé pour lutter contre l'exode rural et ne pas trop dépeupler les zones de mipente et de montagne de l'île (Benoit, 2015).

À cette dichotomie «Hauts-Bas» se superpose un autre découpage territorial historique impulsé par la Compagnie des Indes orientales durant la première moitié du dix-huitième siècle par l'attribution de concessions «du battant des lames au sommet des montagnes». Les terres attribuées aux colons étaient ainsi des lanières allant du bord de la mer à l'intérieur montagneux de l'île (Dalama, 2005). C'est sur la base de ce découpage initial des concessions que l'actuelle répartition spatiale des Réunionnais s'est élaborée ainsi que la création des paroisses ellesmêmes transformées en communes lors de la Révolution française. On retrouve aujourd'hui cette structure spatiale dans le découpage communal (Carte 1).

L'imbrication du social et du spatial à La Réunion est d'autant plus importante que ce département est l'un des plus pauvres de France avec La Guyane et Mayotte. À La Réunion, $42 \%$ de la population vit en 2010 sous le seuil de pauvreté monétaire, c'est-à-dire avec moins de 945 euros par mois par unité de consommation (Insee, 2013b). La Réunion est aussi, et de manière liée au constat précédent, le département le plus inégalitaire de France sur la base des revenus fiscaux de 2011. Ainsi, 
l'indice de Gini qui mesure la dispersion de ces revenus ${ }^{3}$ y est de 0,53 contre 0,50 à Paris et 0,47 en Martinique tandis que $90 \%$ des départements de métropole arborent un indice inférieur à 0,38 (Barnier, 2013). On retrouve ces mêmes résultats au niveau des communes réunionnaises puisque les deux tiers d'entre elles (L'Étang-Salé, Saint-Denis, Saint-Paul, Sainte-Suzanne, Saint-Leu, Le Tampon, Saint-Pierre, SaintAndré, Saint-Benoît, Saint-Joseph, Saint-Louis, Cilaos, Le Port, Salazie, Sainte-Rose) ont des indices de Gini supérieurs à 0,50.

\section{CARTE 1 Les 24 communes de La Réunion}

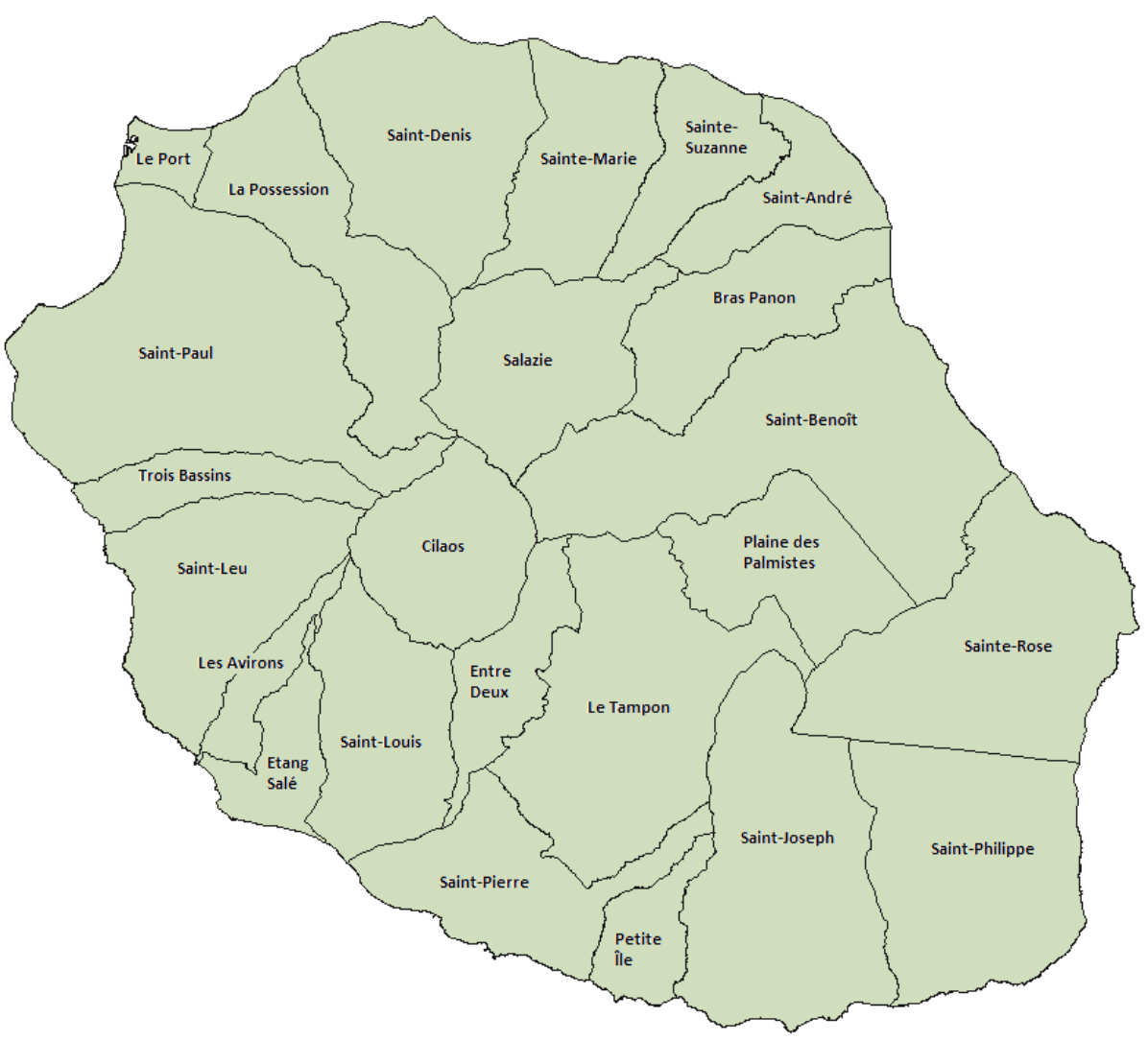

Source : Base de données territorialisées, Caisse d'Allocations Familiales de La Réunion, www.caf974bdt.fr/view/territoires.php?idart=-1\&idrub=22.

3. L'indice de Gini est distribué entre 0 et 1 . Plus il est proche de 1, plus la distribution est inégalitaire. 
Pour mieux comprendre les enjeux du vieillissement de la population et le rôle des politiques publiques à La Réunion en ce domaine, l'ensemble des caractéristiques évoquées ci-dessus incitent donc à adopter une échelle infra-territoriale et notamment communale.

\section{Le vieillissement comparatif de la population à l'échelle communale}

Au début des années 1950, l'indice synthétique de fécondité des femmes réunionnaises est de sept enfants par femme. Une transition démographique, impulsée par le développement et la modernisation socioéconomique, a amené cet indicateur à 2,8 enfants par femme en l'espace de trente ans (Sandron, 2007). Au milieu des années 1990, l'indice synthétique de fécondité atteint un minimum de 2,3 enfants par femme. Depuis 1996, il oscille entre 2,3 et 2,5 enfants par femme (Insee, 2013c). Cette dynamique démographique couplée à une baisse importante de la mortalité a été à l'origine d'une transition démographique très courte, l'une des plus rapides au monde. L'originalité du modèle réunionnais est le maintien, depuis une vingtaine d'années, de l'indice synthétique de fécondité à un niveau relativement élevé, au-dessus du seuil de renouvellement de la population. La conséquence directe de ce constat est le maintien d'une croissance naturelle élevée avec des taux respectifs annuels moyens de $1,6 \%, 1,4 \%$ et $1,3 \%$ respectivement pour les périodes 1990-1999, 1999-2006 et 2006-2011 (Insee, 2014b). Pour ces mêmes périodes, en tenant compte du solde migratoire, les taux de croissance annuels moyens de la population sont respectivement de 1,9\%, 1,5\% et $1,2 \%$.

Ce schéma démographique implique que le département de La Réunion est encore «jeune». En 2013, les moins de vingt ans représentent plus du tiers de la population réunionnaise alors qu'ils sont moins du quart en France métropolitaine (Insee, 2014b). En outre, avec un taux de chômage de $59 \%$ pour les 15-24 ans (Insee, 2014c), cette population occupe une place importante dans l'espace social et politique de La Réunion. Les politiques publiques y consacrent beaucoup d'énergie et se doivent d'innover face à la diversité et la complexité des situations relevées sur le territoire réunionnais. Selon Morel et Maire (2014, p. 119), «les environnements familiaux, sociaux, communautaires, mais aussi territoriaux constituent autant de vecteurs d'inégalités et de clivages sur l'île, et ils éclatent la jeunesse réunionnaise en une mosaïque de conditions et de trajectoires différenciées». Cette préoccupation pour la jeunesse réunionnaise n'est pas récente et si l'on y rajoute une mobilisation politique 
pour faire face à un chômage récurrent important, on perçoit que l'intérêt des politiques publiques pour la problématique du vieillissement de la population est moindre.

L'augmentation croissante et rapide de la proportion des personnes âgées de soixante ans et plus dans la population réunionnaise est pourtant inscrite dans la baisse rapide de la fécondité et de la mortalité à partir des années 1960. Le vieillissement démographique de la population est ainsi apparu dès la décennie 1970, mais avec l'arrivée à l'âge de la retraite des générations nombreuses nées après la Seconde Guerre mondiale, c'est au cours des prochaines années que ce phénomène sera le plus intense. Les personnes âgées de soixante ans et plus qui représentent $13,6 \%$ de la population totale en 2013 seront 25,8 \% en 2040 selon les projections centrales de l'Insee (2014b). L'âge moyen de la population passera de 33 ans à 40 ans durant cette même période. La structure de la population réunionnaise sera similaire en 2040 à celle de la France métropolitaine de 2012 avec une proportion similaire de jeunes de moins de vingt ans et de personnes âgées de soixante ans et plus. Alors que le territoire réunionnais avait accueilli 52700 personnes supplémentaires âgées de soixante ans et plus entre 2000 et 2015, ce sont plus de 100000 personnes âgées supplémentaires entre 2015 et 2030 qui devront être prises en compte, indiquant par là-même les efforts nécessaires en termes de politiques publiques pour promouvoir le bienvieillir de ces personnes.

Ces changements démographiques ne s'opèrent pas de manière homothétique dans toutes les communes de l'île de La Réunion. Ainsi, la croissance démographique telle que mesurée au cours de la période 20062011 est négative pour trois communes (Sainte-Marie, Le Port, Cilaos), tandis que d'autres affichent un gain de population allant jusque 3,5\% par an (La Plaine-des-Palmistes) (Carte 2). S'il ne joue que faiblement dans la croissance démographique globale de l'île, le solde migratoire a un rôle plus important dans la redistribution de la population par commune. En effet, l'accroissement de la population dû au solde migratoire est de l'ordre de $2 \%$ par an pour les communes de La Plaine-des-Palmistes, La Possession et Les Avirons tandis que d'autres connaissent des taux proches de - $2 \%$ (Le Port, Sainte-Marie). Globalement, la croissance de la population est légèrement plus forte entre 2006 et 2011 dans 
les microrégions ${ }^{4}$ de l'Est et du Sud $(1,4 \%)$ que dans celles de l'Ouest et du Nord (respectivement 1,2\% et 0,8\%). Le coût du foncier pourrait ainsi rendre ces dernières moins attractives (Insee, 2014b).

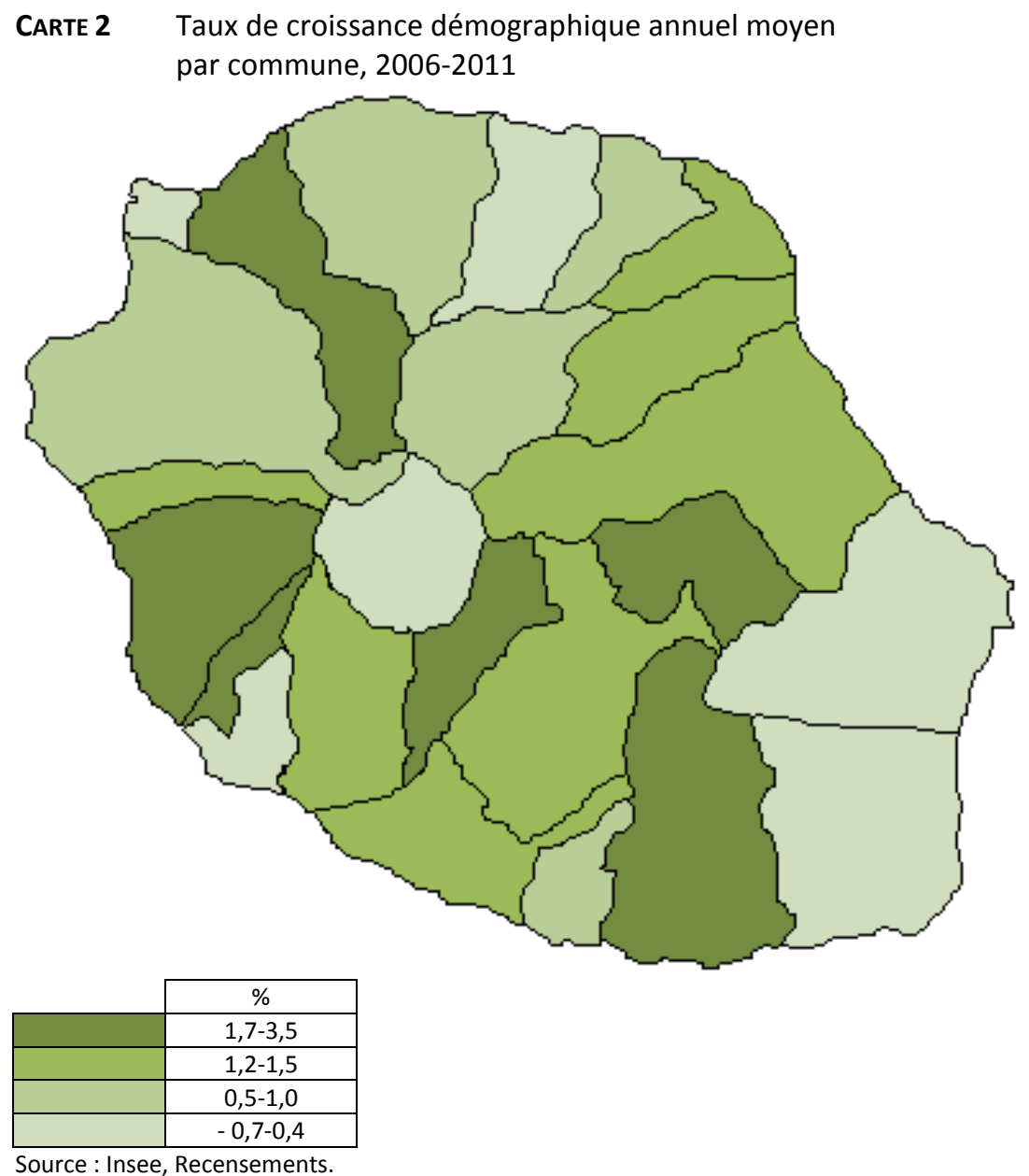

Au niveau communal, la proportion de personnes âgées de soixante ans et plus affiche quant à elle des variations importantes, de $10,3 \%$ à La

4. Région Nord : Saint-Denis, Sainte-Marie, Sainte-Suzanne ; Région Est : Bras-Panon, Saint-André, Saint-Benoît, Plaine-des-Palmistes, Sainte-Rose, Salazie ; Région Ouest : Le Port, La Possession, Saint-Leu, Saint-Paul, Trois-Bassins ; Région Sud: Les Avirons, L'Entre-Deux, L'Étang-Salé, Petite-île, Saint-Joseph, Saint-Louis, Saint-Pierre, Saint-Philippe, Le Tampon, Cilaos. 
Possession à 17,2 \% à Petite-île en 2012 (Carte 3). Les plus fortes proportions de personnes âgées de soixante et plus se retrouvent dans les communes enclavées et des Hauts (Petite-île, Cilaos, Salazie, La Plainedes-Palmistes) ainsi que dans deux communes du Sud (Entre-Deux, Saint-Joseph), avec des taux allant de 15 à $17 \%$. À l'autre extrémité, on trouve des communes comptant moins de $12 \%$ de personnes âgées de soixante ans et plus et qui forment un croissant du Nord-Ouest à l'Est du Département allant de La Possession à Saint-Benoît, exception faite du chef-lieu Saint-Denis (Sainte-Suzanne, La Possession, Saint-André, BrasPanon, Sainte-Marie, Saint-Benoît).

CARTE 3 Proportion de personnes âgées de 60 ans et plus en 2012

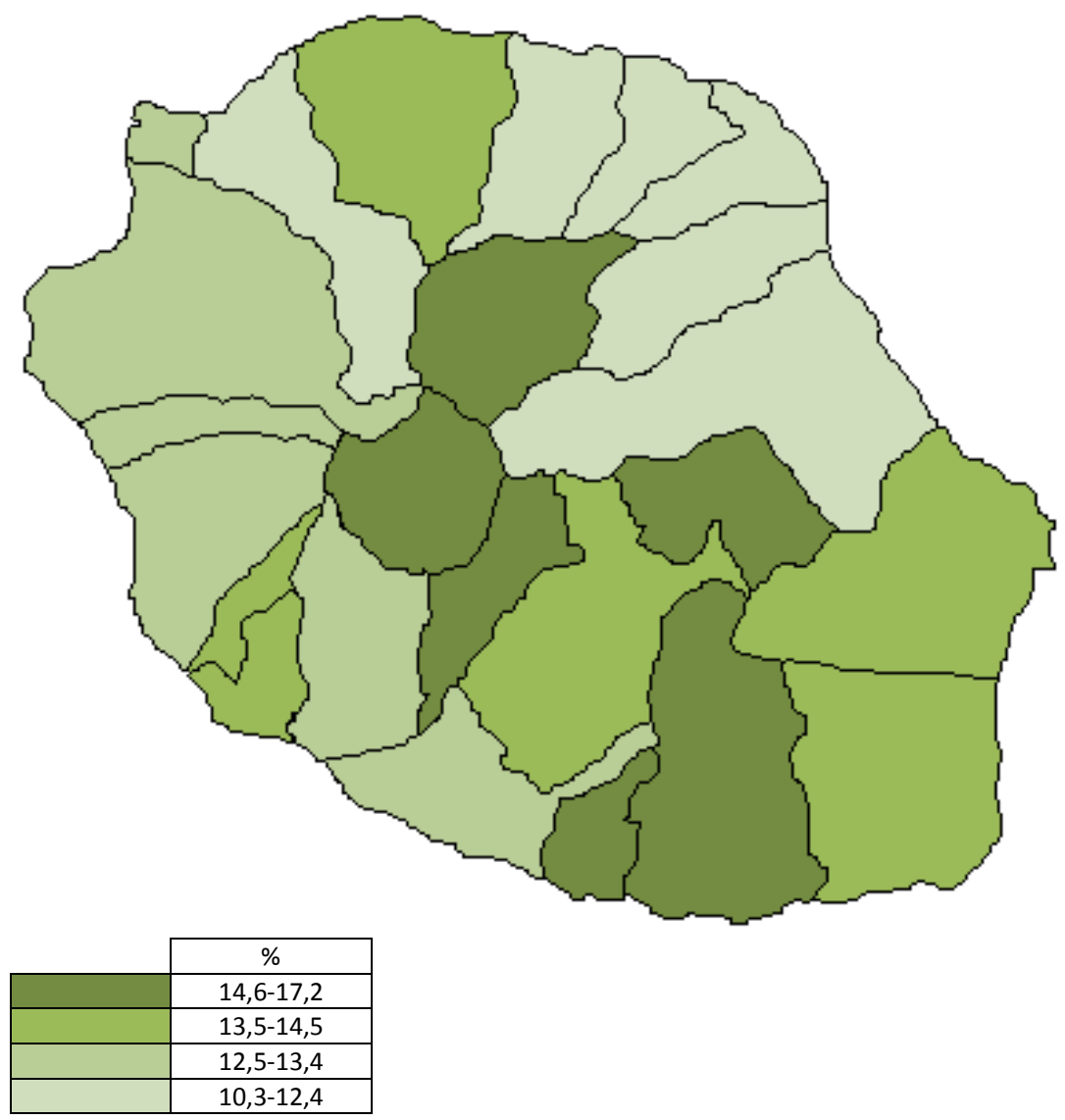

Source : Insee, Recensements.

Avec l'allongement de l'espérance de vie, le vieillissement de la population se fait aussi par le haut de la pyramide des âges, avec un nombre 
toujours croissant de personnes âgées de 75 ans et plus. À l'horizon 2030 , les projections de l'Insee indiquent qu'au sein de la population des soixante ans et plus, ce sont les personnes âgées de 75 ans et plus qui connaîtront la progression la plus forte (Insee, 2014a). Or, il s'agit là d'un âge où la perte d'autonomie devient plus importante. Là encore, l'écart de la distribution entre les communes est significatif puisque les proportions de personnes âgées de 75 ans et plus varient de 2,5\% à La Possession à 5,6\% à l'Entre-Deux. La répartition des personnes âgées de 75 ans et plus est similaire à celle des personnes âgées de soixante ans et plus, indiquant ainsi la stabilité résidentielle des retraités (Carte 4).

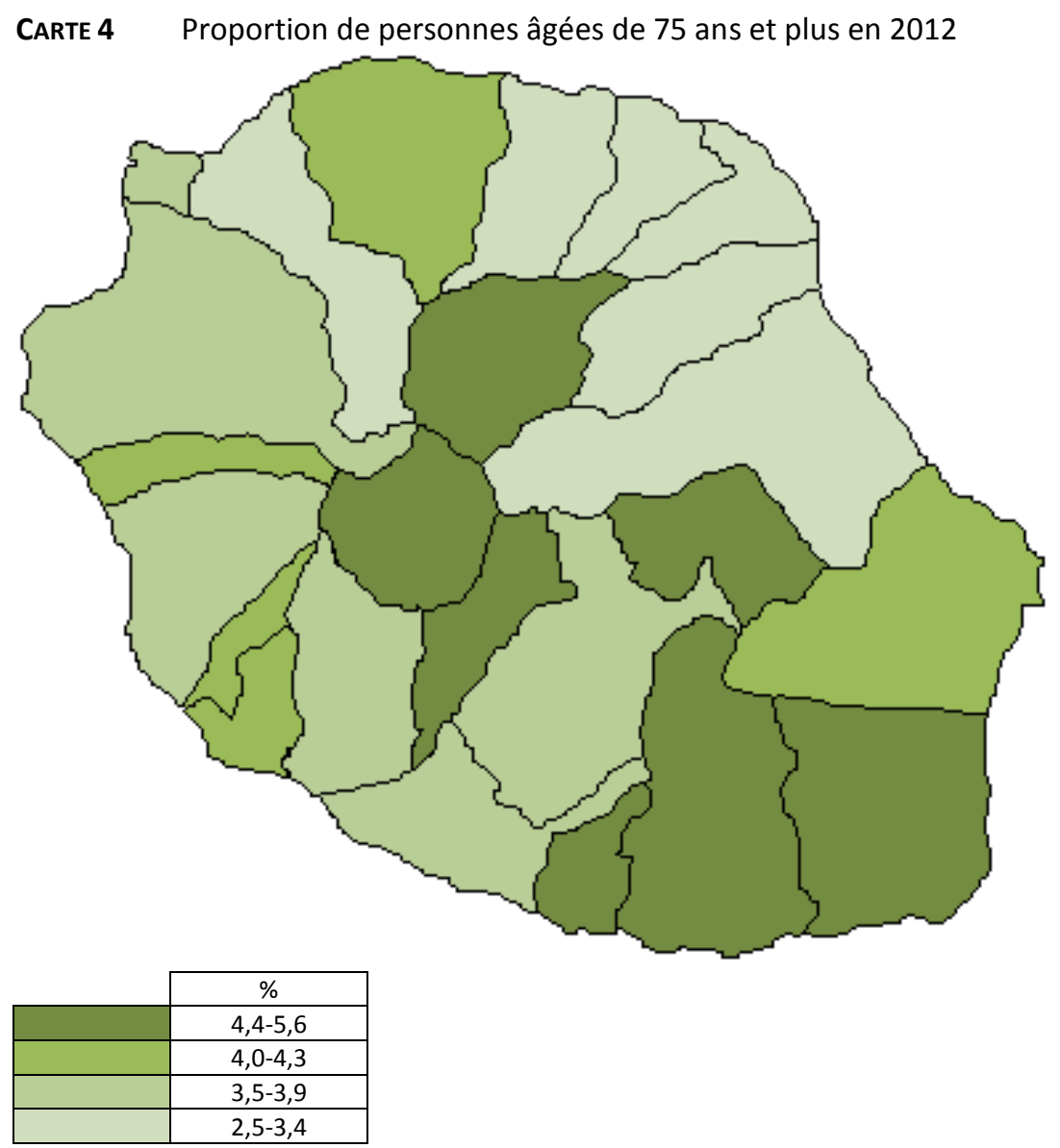

Source : Insee, Recensements. 
CARTE 5 Proportion d'allocataires du minimum vieillesse parmi les personnes âgées de 65 ans et plus en 2008

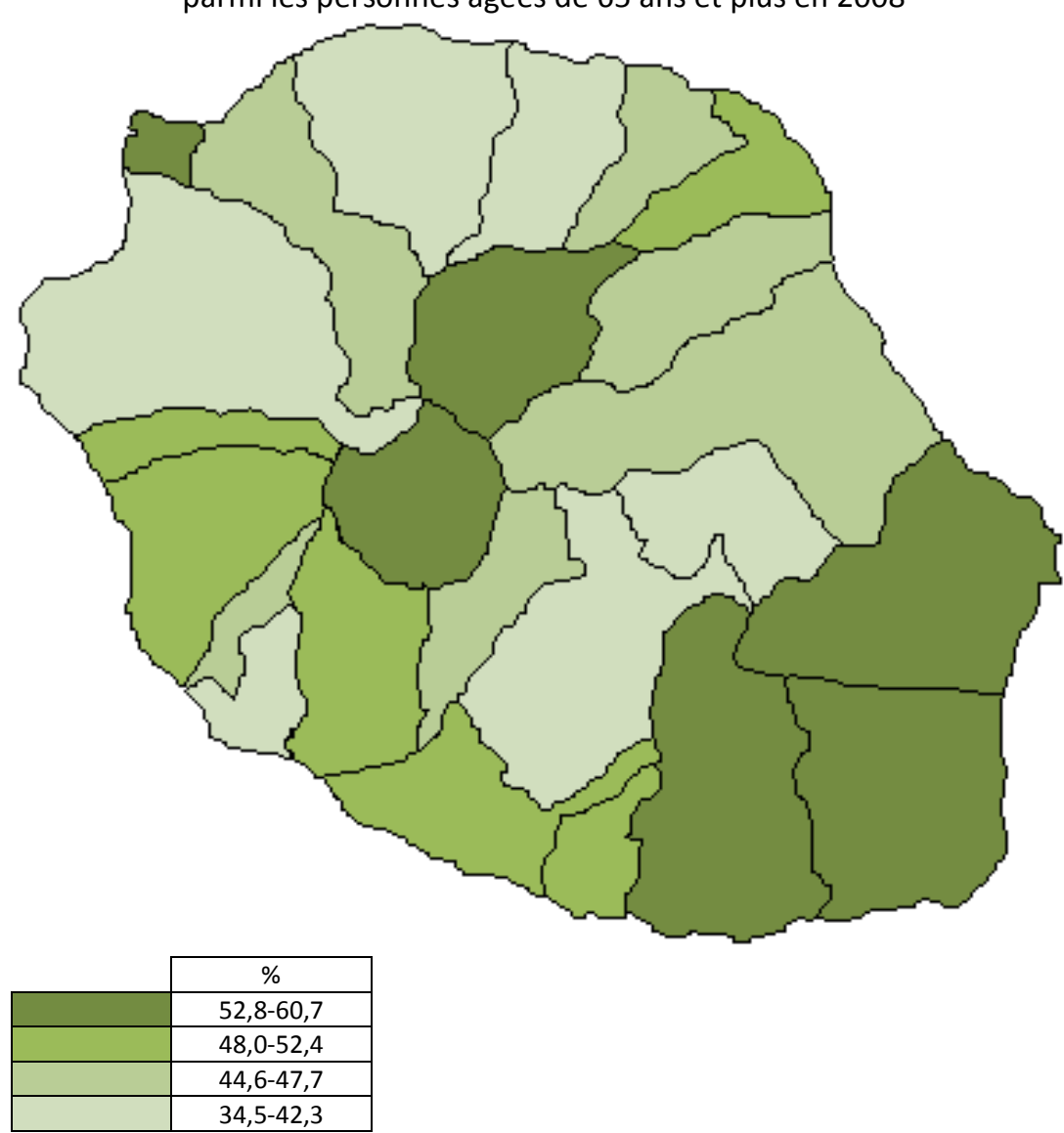

Source : Insee, CGSS, Saspa, MSA, Cavimac, RSI.

Enfin, pour mieux cerner le niveau de vie des personnes âgées en fonction de leur localisation, la proportion d'allocataires du minimum vieillesse fournit un indicateur intéressant. Le minimum vieillesse est en effet une allocation différentielle non contributive qui permet à toute personne âgée de 65 ans et plus de bénéficier d'un revenu minimum de retraite fixé à 633 euros par mois au 1er avril 2008, année de référence des statistiques en notre possession. À La Réunion, $45 \%$ des personnes âgées de 65 ans et plus sont allocataires du minimum vieillesse en 2008, ce qui est considérable en comparaison du chiffre de $5,4 \%$ pour la France métropolitaine (Insee, 2011a). Les écarts sont extrêmement marqués en fonction des tranches d'âge concernées puisque la proportion d'allocataires augmente avec l'âge, allant de $28 \%$ pour les 65-69 
ans à $82 \%$ pour les 90 ans et plus. Ce résultat relate clairement le chemin parcouru depuis les années 1960-70 en termes de développement économique, d'accès au marché de l'emploi et de complétude des carrières. En termes d'analyse différentielle selon les microrégions réunionnaises, les données indiquent une plus forte proportion de personnes âgées bénéficiaires du minimum vieillesse dans le Sud, l'Est et l'Ouest, avec des taux de $46 \%$ à $48 \%$, tandis que le Nord se distingue avec $36 \%$ des personnes âgées concernées (Insee, 2011b). Au niveau communal, la distribution spatiale des allocataires du minimum vieillesse reproduit celle de la précarité de la population et indique des écarts importants entre les cirques de Cilaos et de Salazie autour de $60 \%$ et le chef-lieu Saint-Denis avec $35 \%$ (Carte 5).

\section{Les différentes échelles de l'offre sanitaire, médico-sociale et sociale}

L'offre sanitaire, médico-sociale et sociale sur un territoire est liée à l'effectif de personnes âgées y résidant. Le nombre de personnes âgées est par exemple déterminant pour l'obtention de lits supplémentaires dans un service hospitalier, pour l'ouverture d'une nouvelle structure médico-sociale ou encore pour les montants alloués aux aides sociales ${ }^{5}$. Les communes caractérisées par de forts effectifs de personnes âgées de soixante ans et plus sont logiquement les plus grandes communes de l'île (Saint-Denis, Saint-Paul, Saint-Louis, Saint-Pierre, Le Tampon, SaintAndré, Saint-Joseph) et situées en majorité au Sud (Carte 6). Au total, $38 \%$ des personnes âgées de soixante ans et plus vivent dans le Sud contre $25 \%$ dans le Nord, $24 \%$ dans l'Ouest et $13 \%$ dans l'Est.

Les communes centrales des Hauts (Cilaos, Salazie, Plaine-des-Palmistes, Entre-Deux) qui présentent des proportions de personnes âgées importantes (Carte 3) ne comptent en revanche en termes d'effectif qu'un nombre limité de personnes âgées. Ces quatre communes comptabili-

5. «Les montants de ces dotations sont fixés en fonction des besoins des personnes handicapées et âgées dépendantes, tels qu'ils résultent des programmes interdépartementaux mentionnés à l'article L. 312-5-1, et des priorités définies au niveau national en matière d'accompagnement des personnes handicapées et des personnes âgées. Ils intègrent l'objectif de réduction progressive des inégalités dans l'allocation des ressources entre régions et l'objectif de réduction des inégalités dans l'allocation de ressources entre établissements et services relevant de mêmes catégories, et peuvent à ce titre prendre en compte l'activité et le coût moyen des établissements et services» (Article L314-3 du Code de l'action sociale et des familles). 
sent en effet à elles quatre autant de personnes de soixante ans et plus qu'une commune de taille moyenne comme Sainte-Marie. Ce faible effectif explique en partie que ces zones soient assez faiblement couvertes par l'offre globale aux personnes âgées sans négliger, bien sûr, le caractère enclavé de ces communes.

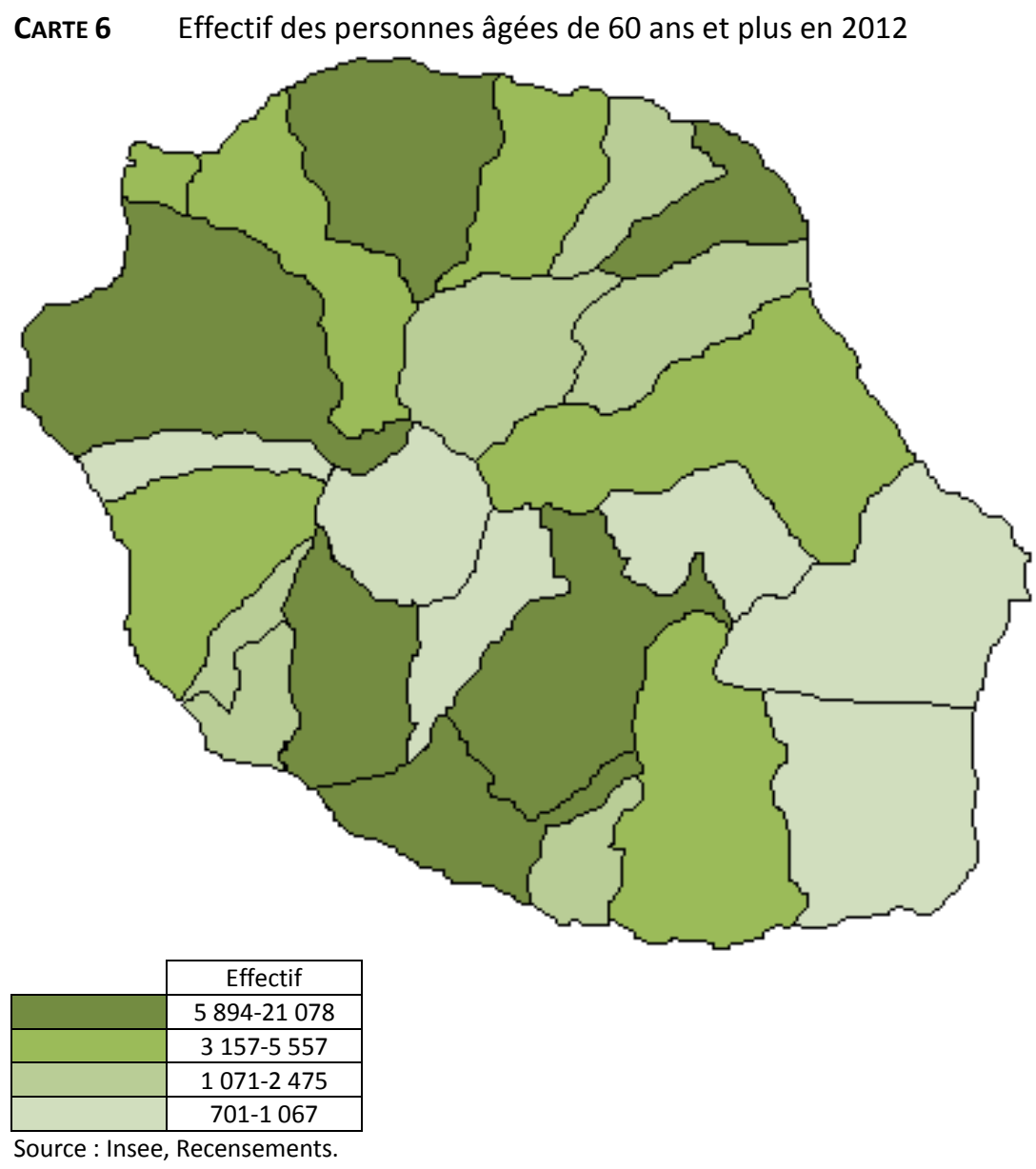

L'écosystème de la prise en charge des personnes âgées, notamment en perte d'autonomie, est caractérisé par un maillage complexe d'institutions, d'organisations et de structures relevant de sphères différentes et plus ou moins hermétiques les unes par rapport aux autres. Liés à des considérations professionnelles, règlementaires ou géographiques (Grenier, Guitton-Philippe, 2011), ces cloisonnements observés dans le domaine sanitaire, médico-social et social sont encore plus visibles lors- 
qu'on s'intéresse à la distribution et à la répartition de l'offre sur un territoire donné. Les échelles différentes et non systématiquement communicantes de ces dispositifs peuvent elles-mêmes être démultipliées selon que l'on se place au niveau décisionnel et gestionnaire ou au niveau de l'action et de ses déclinaisons locales. Ainsi, on distingue l'échelle régionale pour les grandes orientations du sanitaire et du médico-social, l'échelle départementale pour les orientations du médico-social et de la politique sociale, l'échelle des Territoires de santé pour la mise en œuvre de la politique de santé, l'échelle des arrondissements pour l'action sociale du département et de la Sécurité Sociale et l'échelle communale pour l'action sociale des Centres Communaux d'Action Sociale (CCAS). Selon Tonnelier (2010, p. 45), «pour les territoires gérontologiques, les contraintes sont le financement, le rôle de l'État et des départements, le défi l'articulation entre la décentralisation et l'État central». Cette articulation est cruciale pour la cohérence d'une offre sanitaire, médico-sociale et sociale adaptée aux besoins des personnes âgées sur un territoire.

Les filières gériatriques à La Réunion se sont développées dans la mouvance du Plan national Solidarité Grand âge 2007-2012 dont l'objet était d'«assurer une continuité entre le domicile, l'établissement médicosocial et l'établissement de santé» (Jeandel, Vigouroux, 2015, p. 82 ; CHR Réunion, 2009). Elles se sont structurées en premier lieu au sein du Centre Hospitalier Universitaire qui comprend deux pôles principaux, un au Nord à Saint-Denis (Unité de Soins de Longue Durée, Court Séjour de Gériatrie, Équipe Mobile de Gériatrie), et l'autre au Sud réparti sur différents sites, à Saint-Pierre (Unité de Soins de Longue Durée, urgences, Équipe Mobile de Gériatrie), à Saint-Joseph (Court Séjour de Gériatrie, Hôpital de jour) et au Tampon (Unité Cognitivo-Comportementale spécialisée Alzheimer). Ces deux filières comprennent également des partenariats avec des Établissements d'Hébergement pour Personnes âgées dépendantes (EHPAD) et des Structures de Soins de Suite et de Réadaptation au Nord comme au Sud. Chacune de ces filières compte une consultation mémoire labellisée au sein du service de gériatrie dans le Sud et adossée au service de neurologie dans le Nord. En plus du Centre Hospitalier Universitaire, il existe deux groupes hospitaliers, un dans l'Ouest à Saint-Paul et un autre dans l'Est à Saint-André et Saint-Benoît. Ils comprennent tous les deux un service de gériatrie, une Équipe Mobile de Gériatrie et une consultation mémoire. L'offre sanitaire se complète par deux organismes d'Hospitalisation à Domicile, quatre Services de Soins Infirmiers à Domicile et deux équipes Spécialisées Alzheimer qui, contrairement au reste de l'offre sanitaire hospitalière, ne répondent pas à une logique de Territoire de santé mais à une approche globale du terri- 
toire avec des découpages spécifiques à chaque structure. L'offre sanitaire hospitalière est donc encore fortement polarisée entre le Nord et le Sud laissant certaines zones et principalement dans les Hauts dépourvues de telles structures. À l'heure des regroupements et des restructurations, ce constat est d'ailleurs courant pour les communes et territoires enclavés et de faible densité (Bontron, 2013).

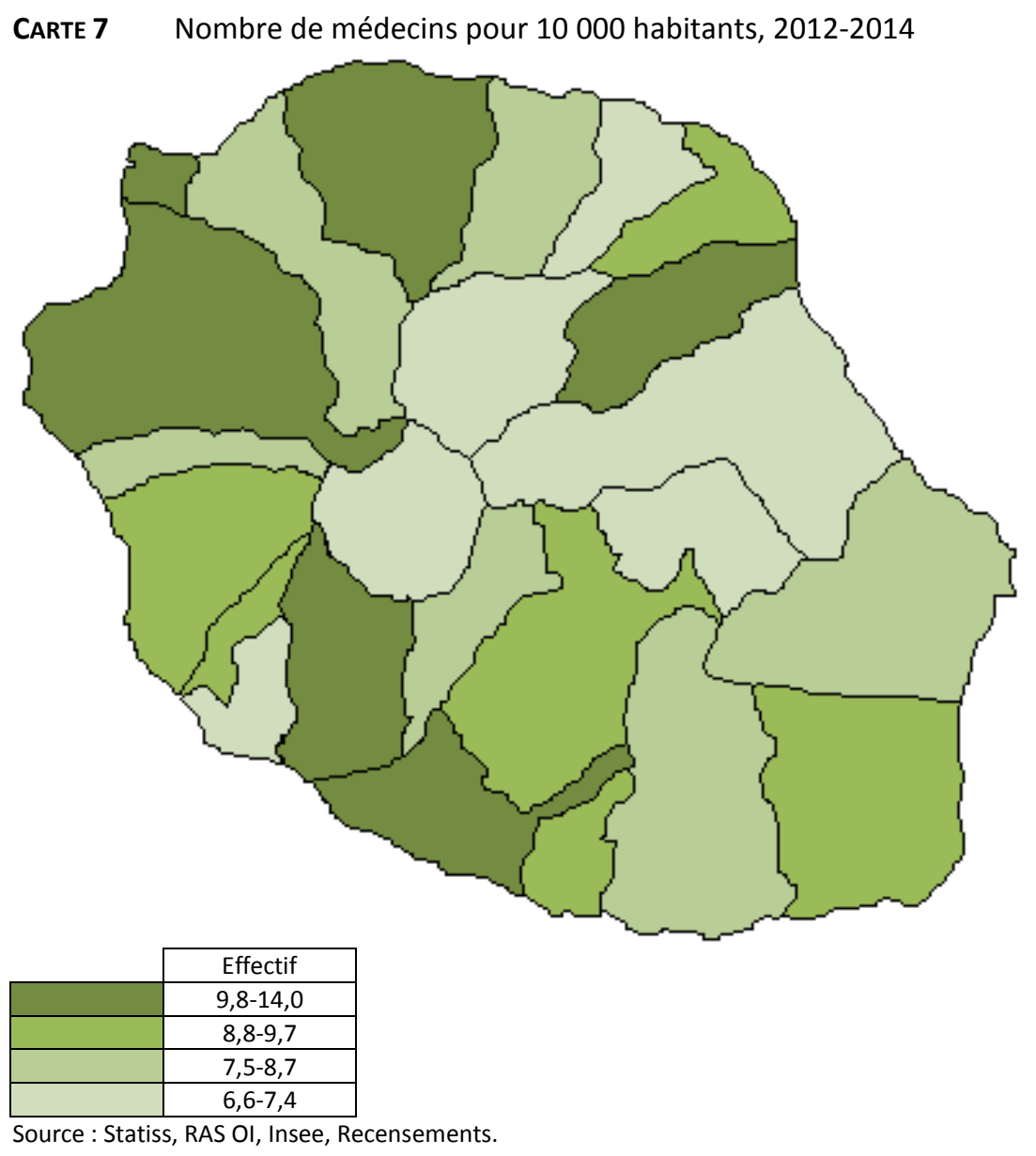


CARTE 8 Nombre d'orthophonistes pour 10000 habitants, 2012-2014

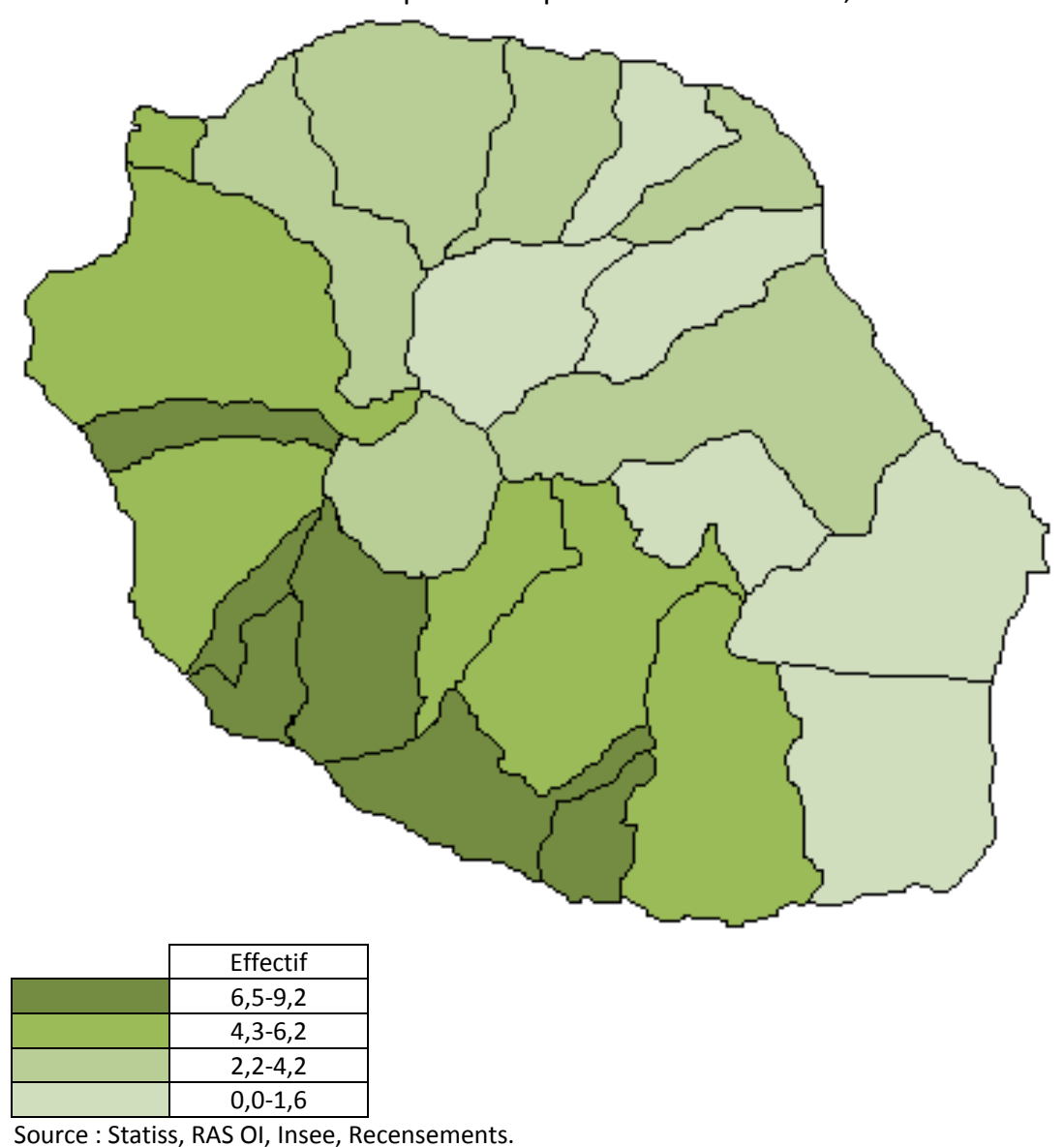

En ville, les densités de professionnels médicaux (médecins généralistes et spécialistes) et paramédicaux (infirmiers, kinésithérapeutes, orthophonistes) libéraux intervenant fréquemment auprès des personnes âgées ne sont pas très éloignées de celles observées en France métropolitaine. Cependant, ces professionnels sont inégalement répartis sur le territoire et leur répartition a plutôt tendance à creuser encore davantage les écarts entre les communes du point de vue de l'offre sanitaire. Les médecins libéraux sont ainsi relativement peu nombreux dans les communes des Hauts déjà citées (Cilaos, Salazie, Plaine-des-Palmistes) et dans quelques communes du littoral (Sainte-Suzanne, Étang-Salé, Saint-Benoît) (Carte 7). Dans le traitement de la maladie d'Alzheimer, il est très fréquemment prescrit des séances d'orthophonie pour retarder le développement de troubles de la parole (Delieutraz et al., 2013). Or, 
les communes des Hauts (Salazie, Plaine-des-Palmistes) pâtissent du manque d'orthophonistes ainsi que certaines communes relativement éloignées des centres hospitaliers (Saint-Philippe, Sainte-Rose). Deux zones se dégagent clairement quant à la densité d'orthophonistes sur le territoire : une première zone plutôt dense allant du Nord-Ouest au Sud et une seconde moins bien desservie allant du Sud-Est au Nord et incluant les Hauts (Carte 8).

Dans le cas de la perte d'autonomie de la personne âgée, le maintien à domicile est le mode de prise en charge le plus largement usité à La Réunion. Même en cas de dépendance lourde, ce sont $83 \%$ des personnes âgées qui restent à leur domicile, contre $49 \%$ en métropole (Insee, 2014a). De façon concomitante, La Réunion est un département avec peu de place en structures d'accueil médico-sociales. En effet, au 1er janvier 2013, le taux d'équipement en structures d'hébergement complet (lits maisons de retraite, lits hébergement temporaire, lits Unité de Soins de Longue Durée) pour les personnes âgées de 75 ans et plus est de 51 pour 1000 à La Réunion contre 124 pour 1000 en métropole (ARS, 2014). Il y a six Établissements d'Hébergement pour Personnes Âgées (EHPA) répartis dans cinq communes (Saint-Leu, Saint-Pierre, Saint-Paul, Sainte-Marie et Le Tampon) et il y a seize EHPAD répartis sur onze communes (Insee, 2014a). Le tiers des places en Unité Alzheimer dans les EHPAD sont concentrées dans le Nord. La moitié des communes de l'île ne contiennent aucune structure d'hébergement complet. L'accueil temporaire qu'il soit en accueil de jour ou en places dédiées en EHPAD est, d'une part, très faible et, d'autre part, polarisé au Nord à Saint-Denis et au Sud à Saint-Pierre.

Enfin, les procédures d'aides sociales spécifiques aux personnes âgées (Allocation Personnalisée d'Autonomie, Aide-ménagère et Chèque santé) sont instruites et suivies par les Actions de Santé, réparties dans les quatre arrondissements du Département de La Réunion. C'est par contre au niveau central du département que se gère l'aide aux aidants avec une dispersion géographique importante. C'est aussi à ce niveau que le dispositif de gratuité des transports sur toute l'île est élaboré pour les personnes de soixante ans et plus, favorisant ainsi leur accès aux services sur le territoire. Comme nous venons de le voir, certaines offres de soins ou de prise en charge ne sont pas aisément accessibles aux personnes ayant des difficultés à se déplacer et/ou aux personnes vivant dans des zones isolées car montagneuses et enclavées. C'est en revanche au niveau communal que les CCAS instruisent les demandes d'aides légales (c'est-à-dire les aides de l'État déléguées par conventionnement) et les aides extra-légales (facultatives et décidées par le conseil d'admi- 
nistration) très variées sur le territoire. La Caisse Générale de la Sécurité Sociale et les caisses complémentaires de retraites fournissent également des aides sociales à leurs pensionnés autonomes. S'ajoute à cela un tissu associatif assez dense et un réseau structuré d'entreprises et d'associations du Maintien à domicile et du Service à la personne qui interviennent sur l'ensemble du territoire. Finalement, c'est sans doute au niveau de l'offre sociale que les politiques publiques sont les plus complémentaires et les plus couvrantes sur le territoire réunionnais.

\section{Dispositifs publics en faveur des personnes âgées et coordination}

Pour des raisons ayant trait à la complexité de la prise en charge du bien-vieillir dans les politiques publiques et face à la volonté d'individualiser les politiques sociales et gérontologiques, la question du maillage territorial des différentes actions en faveur des personnes âgées est de plus en plus essentielle (Argoud, 2013; Bloch, Hénaut, 2014). Loin d'être exhaustive, la revue de quelques dispositifs impulsés sur le territoire réunionnais va permettre de mieux saisir la double injonction d'une approche socialement et géographiquement ciblée et d'une nécessaire coordination des dispositifs existants.

Certains dispositifs sont de fait et par nature territorialisés. C'est le cas par exemple des expériences de télémédecine qui visent à couvrir et améliorer la couverture sanitaire des zones rurales et isolées. L'enclavement est en effet un facteur important dans les inégalités de l'accès aux soins et le rôle des élus locaux pour trouver des solutions innovantes est moteur pour contrecarrer cet effet «isolement» (Gucher, 2013). Or, le relief montagneux de La Réunion rend d'une acuité prégnante cette problématique, spécialement pour les personnes les plus vulnérables et donc les personnes âgées. Ainsi, Cilaos, commune accessible par une route de 400 virages parfois fermée car sujette aux éboulis, et Mafate, ensemble de villages inaccessibles autrement que par des sentiers pédestres ou par hélicoptère, en sont les incarnations les plus frappantes. À Mafate, peuplé d'environ 750 habitants, aucun médecin n'assure de manière permanente les soins. C'est pourquoi deux expériences de télémédecine ont été initiées, en 2004 à Cilaos et en 2010 à Mafate, dans le cadre de téléconsultations et de régulation des urgences médicales (ARS, 2012). Au-delà de leur intérêt direct pour les populations cibles, ces initiatives localisées sont intéressantes aussi pour les retombées qu'elles peuvent générer de manière plus large sur le territoire. Dans le cas présent, selon Béguin et Volia (2014), l'application de ces outils de 
télémédecine nécessite une réorganisation des pratiques de soins et de coordination des professionnels. Ces nouvelles formes de coopération peuvent ainsi servir de modèle ou d'expérimentation sur le reste du territoire.

Un autre dispositif particulièrement développé à l'île de La Réunion est celui des familles d'accueil pour personnes âgées. Selon le site internet du Conseil Départemental de La Réunion6 ${ }^{6}$

\begin{abstract}
«L'accueil familial est une solution intermédiaire entre le maintien à domicile et l'hébergement en établissement. Ce mode d'accueil peut être proposé aux personnes qui ne souhaitent ou qui ne peuvent plus vivre seules à leur domicile et qui privilégient un cadre de vie familial à un hébergement en établissement. Cette formule originale qui met fin à l'isolement d'un public vulnérable constitue une priorité du Conseil Départemental dans sa politique en faveur des personnes âgées et/ou handicapées adultes».
\end{abstract}

L'accueillant familial, après avoir reçu un agrément, peut héberger une à trois personnes âgées de soixante ans et plus moyennant rémunération. $\mathrm{Au}$ niveau de la France entière, ce sont 9742 accueillants agréés qui sont recensés au 31 décembre 2013, avec des écarts entre départements allant de 2 à 507 accueillants et un ratio moyen de 1,43 personne accueillie par accueillant (Horel, 2015). Ces chiffres concernent à la fois les personnes handicapées et les personnes âgées, sachant que la proportion de personnes âgées accueillies est légèrement inférieure à celle des personnes handicapées ( $46 \%$ vs $54 \%$ ). À La Réunion, la place des familles d'accueil est importante et se situe très largement au-dessus de la moyenne nationale avec 489 accueillants familiaux et 779 personnes accueillies en janvier 20147. Contrairement à la France métropolitaine, la progression de l'accueil familial à La Réunion est élevée puisque le nombre de places potentielles a doublé entre 2004 et 2012 (Département de La Réunion, 2012). Cependant, la répartition spatiale des familles d'accueil sur le territoire est très hétérogène puisque la microrégion Sud en compte $60 \%$ sur son seul territoire, les microrégions Est et Ouest chacune $15 \%$ et la microrégion Nord seulement $10 \%$ (Département de La Réunion, 2012). Dans la mesure où la mise en place et le suivi d'un tel dispositif nécessitent des opérations de contrôle, de formation, d'information, de coordination des professionnels du social et du médico-social autour des familles et des personnes âgées (Guillaumin-Laborie, 2006 ; Bentayeb, Poussou, 2003), une étude plus poussée serait donc intéressante pour analyser l'inégale répartition des familles d'accueil réunionnaises à l'aune de ces variables.

6. www.cg974.fr/index.php/L-accueil-familial.html.

7. www.famidac.fr/?-Annuaire-de-1-accueil-familial-202-. 
Les deux dispositifs ci-dessus que sont la télémédecine sur des territoires particuliers et l'accueil familial des personnes âgées à une échelle plus large illustrent chacun la nécessité d'une bonne coordination des acteurs de la santé et du social mais aussi à un niveau supérieur la nécessité d'articuler entre eux l'ensemble des dispositifs au service de la promotion du bien-vieillir et de la prise en charge de la perte d'autonomie. En plein développement, ces nouvelles formes de coordination sont elles-mêmes territorialisées, comme en attestent par exemple les récents dispositifs de Méthode d'Action pour l'Intégration des services d'aide et de soin dans le champ de l'Autonomie (MAIA).

Les Maia sont nées au niveau national avec le Plan Alzheimer et maladies apparentées 2008-20128. Il s'agissait dans la mesure $n^{\circ} 4$ qui leur est dédiée de créer les conditions d'une «meilleure articulation entre les structures de soins, d'information et d'accompagnement [pour] permettre de construire un parcours de prise en charge personnalisé pour chaque personne atteinte de la maladie d'Alzheimer». À l'origine, l'acronyme signifiait «Maison pour l'Autonomie et l'Intégration des malades Alzheimer». Après une expérimentation de deux années, en 2011, les Maia étendent leurs prérogatives à un public cible qui est celui des personnes en perte d'autonomie fonctionnelle du fait des pathologies liées à l'âge, public plus large que les seules personnes atteintes de la maladie d'Alzheimer. Selon Dupont (2012, p. 180) :

«Les Maia ne sont pas de nouvelles structures qui viendraient se superposer aux autres. Elles n'ont pas non plus pour objet de se substituer à l'existant, mais bien au contraire de s'appuyer sur les ressources du territoire en matière d'accueil, d'orientation et de coordination. Maia est une méthode pour conduire des porteurs identifiés de la coordination (réseaux de santé, Clic, conseils généraux...) vers une dynamique d'intégration».

À La Réunion, la Maia est portée par l'association France Alzheimer depuis 2012. Il existe une seule Maia pour l'ensemble du département mais le maillage territorial se décompose sur les trois territoires NordEst, Sud et Ouest. C'est à cette échelle qu'interviennent les professionnels que sont les gestionnaires de cas, dont le rôle est d'intervenir auprès des personnes âgées en situation complexe, à travers un ensemble de compétences faisant l'objet en France d'une formation spécifique et réglementaire depuis 2012 :

«Identification du bon niveau d'intervention, évaluation multidimensionnelle standardisée, planification de l'ensemble des aides (soins et services), mise en place du plan, monitorage et réévaluation de celui-ci et réévaluation régulière

8. www.plan-alzheimer.gouv.fr/. 
de l'ensemble des besoins dans un processus continu et de long terme» (Somme et al., 2015, p. 61).

C'est d'ailleurs cette fonction de gestion de cas complexe qui semble la plus importante aujourd'hui dans le dispositif de la Maia de La Réunion. Ce dernier constat est variable d'une Maia à une autre pour l'ensemble du dispositif français, qui avec 300 Maia en $2015^{9}$ connaît des situations très diversifiées. Cette diversité des modalités d'action des Maia en France n'est autre que le reflet de l'hétérogénéité des territoires sur lesquels elles se déploient et de la dynamique locale des dispositifs de coordination (Gand, Periac, 2015).

Un autre dispositif innovant en cours à La Réunion est celui de la Conférence des financeurs élaboré dans le cadre de la loi $n^{\circ}$ 2015-1776 du 28 décembre 2015 relative à l'adaptation de la société au vieillissement et considérée comme un «impératif national et une priorité de l'ensemble des politiques publiques de la Nation» ${ }^{10}$. L'article L.233-1 de cette loi stipule que :

\begin{abstract}
«Dans chaque département, une conférence des financeurs de la prévention de la perte d'autonomie des personnes âgées établit un diagnostic des besoins des personnes âgées de soixante ans et plus résidant sur le territoire départemental, recense les initiatives locales et définit un programme coordonné de financement des actions individuelles et collectives de prévention. Les financements alloués interviennent en complément des prestations légales ou réglementaires. Le diagnostic est établi à partir des besoins recensés, notamment, par le schéma départemental relatif aux personnes en perte d'autonomie [...]».
\end{abstract}

La Réunion a été retenue en 2015 parmi les 26 départements préfigurateurs de la Conférence des financeurs. Le 18 novembre 2015, il a été validé un comité exécutif de cette Conférence composé du Conseil Départemental, de l'Agence de Santé Océan Indien et du Groupement d'Intérêt Économique Vieillissement Actif ${ }^{11}$. Les premières opérations concernent un diagnostic de territoire et des actions de prévention en faveur des personnes âgées et des aidants familiaux. Le programme «Proximité Autonomie et Avancée en Age \& Conférence des Financeurs» lancé en 2015 par le GIE Vieillissement Actif 12 s'inscrit dans ce cadre. Il s'agit d'un appel à projets permettant de prévenir la perte d'autonomie et de maintenir le lien social pour des personnes âgées de 55 ans et plus,

9. www.cnsa.fr/parcours-de-vie/maia.

10. www.legifrance.gouv.fr/eli/loi/2015/12/28/AFSX1404296L/jo/texte.

11. www.cg974.fr/index.php/Le-Departement-1ARS-et-1ARS-OI-et-le-GIE-Vieillissement-Actif-installent-la-Conference-des-financeurs-19-novembre-2015.html.

12. GIE créé en septembre 2014 par la Caisse générale de Sécurité Sociale et le Régime Social des Indépendants. 
vivant à domicile et en risque de fragilité. Les zones prioritaires sont les communes identifiées comme les plus fragiles: Bras-Panon, Cilaos, Le Port, Saint-André, Saint-Benoît, Saint-Joseph, Saint-Louis, Saint-Philippe, Sainte-Rose, Salazie, Trois Bassins. Il s'agit donc d'identifier à la fois des personnes en risque de fragilité et des territoires infra-départementaux défavorisés jouant comme un facteur supplémentaire de la fragilité ou de la pré-fragilité de ses ressortissants âgés.

Ces différentes initiatives en termes de prévention s'inscrivent dans le constat de plus en plus généralisé que nous disposons aujourd'hui de bonnes connaissances sur les méthodes et pratiques garantes d'un bienvieillir, ou tout au moins d'un mieux-vieillir, et que c'est dans leur agencement organisationnel que les pistes doivent être creusées (Forette, 2012 ; Trillard, 2012). La question des échelles et de la réplicabilité de leur mode de coordination et d'intégration au sein des territoires est en ce sens fondamentale.

\section{Conclusion}

L'offre sanitaire, médico-sociale et sociale pour les personnes âgées est caractérisée par l'existence de multiples acteurs pour des interventions multi-échelles sur le territoire. Même si les directives nationales constituent l'armature de l'intervention publique, ce contexte nécessite des initiatives locales permettant de promouvoir des démarches préventives à travers des réponses transversales différenciées selon les territoires et en somme d'adapter la mise en œuvre des politiques publiques à leur contexte socioéconomique et à l'évolution démographique de leur population (Dumont, 2010 ; Aquino, 2012).

La volonté politique d'individualiser l'accompagnement du bien-vieillir passe par une connaissance fine des besoins et des caractéristiques de chaque personne âgée, ce qui, pour l'instant, ne peut être réalisé que dans le cadre d'actions de proximité élaborées le plus souvent au niveau des communes. Cependant, le panel des outils de prévention, des solutions de prise en charge ou des aides diverses est directement fonction de l'offre locale de services et des capacités de financement des personnes, ce qui rend le plus souvent la réalisation effective d'une telle politique problématique, notamment dans les zones enclavées ou isolées (Argoud, 2013).

À La Réunion, la pauvreté, le faible niveau de littératie et d'empowerment des personnes âgées rendent cette considération d'autant plus 
prégnante. En revanche, les solidarités familiales et l'existence d'une coordination informelle gérontologique liée au caractère bien circonscrit du territoire sont des atouts pour offrir des solutions adaptées au souhait des personnes âgées de se maintenir à domicile le plus longtemps possible. De manière générale, selon Goavec et Hoarau (2015, p. 195), «le bon niveau de développement, malgré une vulnérabilité structurelle avérée, montre que l'utilisation des transferts publics associés au statut de territoire ultrapériphérique paraît particulièrement efficace dans le cadre de l'économie réunionnaise». Face aux considérations infra-territoriales évoquées ci-dessus, faire jouer un rôle important aux politiques publiques dans le cadre du vieillissement de la population réunionnaise incite donc à se pencher sur les échelles optimales d'action pour réduire les inégalités d'accès à l'offre sanitaire et sociale tout en gardant une flexibilité dans la définition de ces politiques eu égard aux mutations extrêmement rapides de l'évolution socioéconomique du territoire. Ceci est particulièrement vrai pour les personnes âgées dont les générations à venir auront des niveaux d'éducation et de qualification de plus en plus élevés.

\section{Références}

AQUINo J.-P. (2012), "Vieillissement et politiques publiques», Gérontologie et Société, HS 1, pp. 273-279, https://doi.org/10.3917/gs.hs01.0273.

ARGoud D. (2013), "La prise en compte des nouveaux lieux du vieillir par les politiques publiques françaises», M. MEMBRADO, A. ROuYER (eds), Habiter et Vieillir, Érès Éditions, pp. 213-224.

ARS (2012), Programme des systèmes d'information et de télémédecine de La Réunion et de Mayotte (PSIT), Agence Régionale de Santé Océan Indien, Saint-Denis de La Réunion, $56 \mathrm{p}$.

ARS (2014), STATISS. Statistiques et indicateurs de la Santé et du Social, Agence Régionale de Santé Océan Indien, Saint-Denis de La Réunion, 32 p.

BARNIER M. (2013), «Des départements d'Outre-mer marqués par les difficultés sociales et les inégalités», Compas Études, 9, 8 p.

BÉGUIN M., VolıA D. (2014), «La télémédecine au service de la médecine d’urgence en territoire isolé, exemple de l'hôpital de Cilaos (CHU de la Réunion)", Recherche européenne en télémédecine, 3 (4), p. 192.

BENOIT G. (2015), "Cinquante années de politique agraire et d'aménagement du territoire dans l'île de La Réunion", Annales des Mines - Responsabilité et environnement, 3, pp. 51-55. 
Bentayeb C., Poussou C. (2003), "Travail d'équipe et travail en partenariat», L'accueil familial en revue, 15, pp. 53-58.

BerRut G., GuÉRIN S. (2014), "Aménagement du territoire et vieillissement», Gériatrie et Psychologie Neuropsychiatrie du Vieillissement, 12 (3), pp. 243-246.

Bloch M.-A., HénAut L. (2014), Coordination et parcours. La dynamique du monde sanitaire, social et médico-social, Dunod, Paris, $315 \mathrm{p}$.

Bontron J.-C. (2013), "L'accès aux soins des personnes âgées en milieu rural : problématiques et expériences», Gérontologie et Société, 146, pp. 153-171, https:// doi.org/10.3917/gs.146.0153.

BRIGUguo L. (1995), «Small Island Developing States and their Economic Vulnerabilities», World Development, 23 (9), pp. 1'615-1'632.

CHR Réunion (2009), "La filière gériatrique du CHR. Ces personnes âgées qui ont besoin de nous», Convergence, 5, Centre Hospitalier Régional de La Réunion, pp. 12-18.

Dalama M.-G. (2005), "L'île de la Réunion et le tourisme : d'une île de la désunion à la Réunion des Hauts et Bas», L'Espace Géographique, 34 (4), pp. 342-349, https:// doi.org/10.3917/eg.344.0342.

Delieutraz C., Rocher C., Peuchet V., Debray M. (2013), «Rôle de l'orthophoniste dans la maladie d'Alzheimer: du Centre d'évaluation gériatrique au suivi libéral», Revue de Gériatrie, 38 (9), pp. 667-672.

Département de La Réunion (2012), Schéma départemental d'organisation sociale et médico-sociale. 2013-2017. Personnes âgées, Saint-Denis de La Réunion, 115 p.

Dumont G.-F. (2010), "La géographie des territoires gérontologiques», Gérontologie et Société, 132, pp. 47-62, https://doi.org/10.3917/gs.132.0047.

DUPONT O. (2012), «De l'expérimentation des MAIA à leur déploiement», Gérontologie et Société, HS 1, pp. 79-184, https://doi.org/10.3917/gs.hs01.0179.

ForetTe F. (2012), "Les enjeux de la prévention», Gérontologie et Société, HS 1, pp. 27-40, https://doi.org/10.3917/gs.hs01.0027.

Gand S., Periac E. (2015), "Vers des écosystèmes de services gérontologiques ?", Communication au Congrès IAE, 10-12 juin, Rennes, France, $23 \mathrm{p}$.

Goavec C., Hoarau J.-F. (2015), "Une mesure de la vulnérabilité économique structurelle pour une économie ultrapériphérique européenne : le cas de La Réunion», Géographie, Économie, Société, 17 (2), pp. 177-200, https://doi.org/10.3166/ges. 17.177-200.

Grenier C., Guitton-PhiLIPPE S. (2011), «La question des regroupements/mutualisations dans le champ sanitaire et social : I'institutionnalisation d'un mouvement stratégique ?», Revue Management et Avenir, 47, pp. 99-113, https://doi.org/10. 3917/mav.047.0098. 
GUCHER C. (2013), "Le vieillissement des populations et des territoires au prisme d'une ruralité transformée», Gérontologie et Société, 146, pp. 11-20, https://doi. org/10.3917/gs.146.0011.

Guillaumin-LABORIE J. (2006), «Familles d'accueil de personnes âgées : entre identités et représentations professionnelles», L'accueil Familial en Revue, 16, pp. 44-49.

HoRel C. (2015), L'accueil familial des personnes âgées ou handicapées. État des lieux 2014, Institut de formation de recherche et d'évaluation des pratiques médico-sociales, Paris, $82 \mathrm{p}$.

Insee (2010), "Personnes âgées. Démographie et conditions de vie», Économie de la Réunion, 137, pp. 11-19.

Insee (2011a), "Les allocataires du minimum vieillesse en 2008», Informations Rapides Réunion, 183, $4 \mathrm{p}$.

Insee (2011b), «Minimum vieillesse et niveau de vie des personnes âgées réunionnaises en 2008», Insee Résultats, 41, 25 p.

Insee (2013a), «Des quartiers inégaux face à la précarité», Insee Partenaires, 26, $6 \mathrm{p}$.

Insee (2013b), "Indicateurs sociaux départementaux. Une situation sociale hors norme», Insee Partenaires, 25, 4 p.

Insee (2013c), "Un taux de fécondité toujours élevé qui ne baisse plus», Informations Rapides Réunion, 252, 3 p.

Insee (2014a), «Deux fois plus de personnes âgées dépendantes en 2030 à la Réunion», Insee Partenaires, 29, 6 p.

Insee (2014b), «Tableau économique de La Réunion 2014», Insee Réunion, 169 p.

Insee (2014c), «Enquête Emploi 2013 à La Réunion. Un taux de chômage autour de 29 \% depuis quatre ans», Informations Rapides Réunion, 300, 4 p.

Insee (2015), «Bilan démographique 2013. La croissance de la population ralentit», Insee Flash Réunion, 33, 2 p.

Jeandel C., Vigouroux P. (2015), "Huit recommandations pour adapter la prise en charge des personnes âgées en établissement de santé», Cahiers de l'Année Gérontologique, 7, pp. 78-86, https://doi.org/10.1007/s12612-015-0451-9.

L'HORTY Y. (2014), "La persistance du chômage ultramarin : un problème aux causes multiples», Revue Française des Affaires Sociales, 4, pp. 114-135.

Morel S., MaIRE S. (2014), "La jeunesse à La Réunion, une mise en prospective», Informations Sociales, 186, pp. 118-124.

Rochoux J.-Y. (2004), "Croissance économique et chômage à La Réunion en 2020 : perspectives et politiques», A. MaURIN, J.-G. MontaUban, F. Vellas (eds), L'enjeu du développement économique insulaire, Sedes, Paris, pp. 31-74. 
SANDRON F. (2007), «Dynamique de la population réunionnaise», F. SANDRON (ed), La population réunionnaise. Analyse démographique, Éditions de I'IRD, Institut de Recherche pour le Développement, Paris, pp. 27-41, https://doi.org/10.4000/books. irdeditions.123.

Somme D., Corvol A., Couturier Y., Pimouguet C., Moreau O., Perivier S., Balard F., DE STAMPA M. (2015), «Nouveau champ professionnel en France. Les besoins de formation des gestionnaires de cas», Santé Publique, HS 1, pp. 61-66, https://doi.org/ 10.3917/spub.150.0061.

TONNELIER F. (2010), «Bassins de santé et territoires gérontologiques: limites et frontières», Gérontologie et Société, 132, pp. 35-45, https://doi.org/10.3917/gs. 132.0035.

TriLlard A. (2012), "Penser les mesures de prévention en faveur du 'bien vieillir'», Gérontologie et Société, HS 1, pp. 19-25, https://doi.org/10.3917/gs.hs01.0019. 